\title{
¿Para qué filología? \\ Significación filosófica de la edición Colli-Montinari de la obra de Nietzsche
}

\author{
MARCO PARMEGGIANI
}

La obra de Nietzsche posee unas características muy particulares, que la separan netamente dentro de la historia de la filosofía. Ellas plantean problemas muy complejos de interpretación 1 . Actualmente, cualquier investigación rigurosa sobre pensamiento debe comenzar exponiendo los presupuestos metodológicos adoptados para la interpretación de su obra, justificándolos o sólo indicándolos. Es la cuestión que entre la crítica alemana, y en torno a los Nietzsche-Studien, se ha denominado, nietzscheanamente, Nietzsche-lesen: el problema de 'aprender a leer bien' a Nietzsche. Esta tarea tiene ante sí dos problemas hermenéuticos básicos: la división que presenta su obra en, por una parte libros publicados, y por otra fragmentos póstumos; y el carácter fragmentario de toda su obra.

1 La discusión acerca de los problemas de la interpretación de Nietzsche ocupa un lugar central en la bibliografía especializada actual. Entre los trabajos más importantes cf. E. Biser, «Das Desiderat einer Nietzsche-Hermeneutik. Der Gang der Wirkungsgeschichte», NietzscheStudien, 9 (1980), 1-37; S. L. Gilman, Nietzschean Parody. An introduction to reading Nietzsche, Bonn, Bouvier, 1976; P. Heller, «Multiperspektiven Interpretieren. Bemerkungen zum Thema 'Nietzsche lesen'», Nietzsche-Studien, 13 (1984), 624-642; R. L. Howey, «Some Difficulties about reading Nietzsche», Nietzsche-Studien, 8 (1979), 378-388; F. Kaulbach, Sprachen der ewigen Wiederkebr: die Denksituationen des Philosophen Nietzsche und ibre Sprachstile, Würzburg, Königshausen \& Neumann, 1985; M. Montinari, Nietzsche lesen, Berlin, W. de Gruyter, 1982; y H. J. Schmidt, «Mindestensbedingungen nietzscheadäqueterer Nietzsche-Interpretation oder Versuch einer produktiven Provokation», Nietzsche-Studien, 18 (1989), 440-454. 
El presente estudio dilucidará el primer problema hermenéutico bajo un punto de vista particularmente privilegiado: discutiendo la significación, no sólo filológica, sino filosófica de la edición ColliMontinari.

La importancia de las ediciones críticas corre el riesgo de ser infravalorada en el ámbito académico filosófico. Así ha ocurrido con la primera edición crítica de la obra nietzscheana: la edición preparada por Giorgio Colli y Mazzino Montinari — debido a su muerte prematura, no llegaron a acabarla, y en la actualidad dirigen su continuación Wolfgang Müller-Lauter y Karl Pestalozzi². Entre numerosos historiadores de la filosofía y especialistas, domina la creencia de que, bajo el punto de vista filosófico, la aportación de la nueva edición se resume a dos: la recuperación de numerosos textos inéditos de Nietzsche, y la recuperación en su forma original de textos tergiversados por las ediciones anteriores. El resto es interés filológico. No se le concede mucha importancia a la publicación de los manuscritos en su orden cronológico, cuyo fin era reemplazar la presentación temáticamente sistematizada que ofrecía el libro espurio La voluntad de poder.

Montinari ha demostrado que el motivo que llevó a Nietzsche a no publicar la proyectada obra La voluntad de poder no es ninguna de los que suelen circular en la literatura nietzscheana al uso: no procedió de su fracaso personal en darle forma a un material inmenso, de su incapacidad en darle cohesión para configurar un sistema, pero tampoco fue la locura lo que truncó el proyecto: simplemente Nietzsche renunció a él, y todo lo que tenía que escribir como 'obra capital' quedó plasmado en parte en El crepúsculo de los ídolos, pero sobre todo en $E l$ anticristo. Montinari demuestra con documentos cómo es esta obra todo el Ensayo de una transvaloración de los valores: al final, Nietzsche sólo se quedó con el subtítulo, renunció al título original La voluntad de poder y redujo drásticamente el proyecto original:

«Así, puede concluirse de ello que entre el 26 de agosto y el 3 de septiembre de 1888 ocurrió lo siguiente:

1. Nietzsche renunció a la hasta ahora planeada Voluntad de poder.

2 Cf. W. Müller-Lauter, «Zwischenbilanz. Zur Wieterführung der von Montinari mitbegründeten Nietzsche-Editionen nach 1986», Nietzsche-Studien, 23 (1994), 307-316. 
2. Durante breve tiempo ponderó la posibilidad de editar, como Transvaloración de todos los valores, el material ya redactado en limpio.

3. Sin embargo, se decidió por publicar un 'extracto' de su filosofía.

4. Al extracto le dio el título Ociosidad de un psicólogo (más tarde, Crepúsculo de los ídolos).

5. Así mismo, quitó del extracto los capítulos «Nosotros hiperbóreos», «Con nosotros - contra nosotros», «Conceptos de una religión de la décadence», «Budismo y cristianismo», los cuales, conjuntamente, dieron por resultado 24 parágrafos sobre el cristianismo, acompañados de una introducción («Nosotros hiperbóreos»).

6. La obra capital llevó desde ahora como título: Transvaloración de todos los valores, que provenía del subtítulo de la hasta ahora proyectada Voluntad de poder; fue planeada en cuatro libros; de ellos, el primer libro, El anticristo, ya estaba listo en una tercera parte (precisamente los mencionados primeros venticuatro parágrafos).

[...] [Montinari sigue demostrando, con los esbozos de proyectos literarios y las cartas, que Nietzsche renunció por fin a su obra capital y redujo toda la Transvaloración de todos los valores a su primer libro, El anticristo, y concluye:]

Si en cuanto al contenido la Transvaloración de todos los valores es igual, ciertamente, a la Voluntad de poder; sin embargo, es precisamente por ello su negación literaria. O también: a partir de los apuntes para la Voluntad de poder surgieron el Crepúsculo de los ídolos y El anticristo; el resto es... obra póstuma» ${ }^{3}$.

Sin embargo, muchos especialistas siguen basándose y citando la antigua edición de La voluntad de poder, porque es más cómodo y práctico: en ella el material lo encontramos ya organizado temáticamente, no tenemos que ocuparnos de la fastidiosa tarea de recopilar y clasificar, a lo largo de toda la inmensa obra póstuma nietzscheana, los textos que nos interesan (así hacen con frecuencia los especialistas

3 M. Montinari, «Nietzsches Nachlaß 1885-1888 und der "Wille zur Macht"», en F. Nietzsche, Sämtliche Werke. Kritische Studienausgabe, ed. G. Colli y M. Montinari, Berlin/ München, Gruyter/dtv, 19882, vol. 14, pp. 398-400 (en adelante citada con las siglas KSA seguidas del número de tomo).. 
anglófonos sin preocuparse por justificarlo mucho, y en particular uno tan reputado como Schacht $\left.{ }^{4}\right)$.

En nuestra opinión la aportación de la edición Colli-Montinari, respecto a los manuscritos nietzscheanos, no es sólo filológica sino estrictamente filosófica: comprendida en su justa dimensión, ha provocado un cambio en la manera misma de interpretar filosóficamente el pensamiento de Nietzsche. La contribución de la edición ColliMontinari es filosófica en puridad, a lo menos por los siguientes aspectos.

\section{Nivel hermenéutico De LOS textos}

La edición Colli-Montinari ha venido a demostrar que no podemos acercarnos a los distintos textos póstumos con la misma actitud hermenéutica. Anteriormente, al estar reunidos en un libro proyectado, daban la impresión que podían e incluso debían ser interpretados todos de la misma manera y dándoles la misma importancia en cuanto a la operación de lectura, con las únicas diferencias en su extensión o interés. Ahora, la edición Colli-Montinari restituye los manuscritos en su forma original y en su orden cronológico, y a través de un análisis de crítica textual los especialistas van descubriendo que no todos tienen la misma importancia como expresión del pensamiento del autor, de manera que no nos podemos acercar a todos de la misma manera. Los textos contenidos en los manuscritos son de tipo muy diverso. Para su interpretación es imprescindible atender a su variedad tipológica; no es lícito utilizarlos desenfadadamente para apoyar nuestras suposiciones más caprichosas. Hay que distinguir entre:

1) Apuntes descartados completamente. Este trabajo no ha sido realizado casi en lo más mínimo, sino que se han considerado ingenuamente como expresión fiel de sus ideas todos los textos de los manuscritos. Para determinar estos textos sólo cabe recu-

4 Cf. R. Schacht, Nietzsche, London, Routledge, 1992, pp. xii-xiii: «I have sought to direct the attention of resaders to texts indicative of his philosophical thinking which they might reasonably be expected to have read or to be able to read themselves. I take The Will to Power to be such a text (or collection of texts). I leave to others with more narrowly scholarly interests the labor of considering what refinements of interpretation might be suggested by other material in his notebooks». 
rrir a indicios claros e inequívocos: tachaduras, indicaciones expresas por parte de Nietzsche. Una vez individuados, serían muy útiles para cerciorarse de qué planteamientos, ideas o tesis Nietzsche ha desechado y perfilar así más minuciosamente sus planteamientos e ideas propios.

2) Apuntes preparatorios y superados en elaboraciones posteriores. Individuados exhaustivamente por el aparato crítico de la edición en curso (Nachbericht). La determinación de este tipo de textos sirve para entender e interpretar las versiones posteriores y definitivas más arduas, o para perfilar nuestra interpretación observando lo descartado, los cambios formales y la génesis. Desde luego, en ningún caso deben ser tomados por sí mismos, como se hace con tanta frecuencia.

3) Resúmenes, paráfrasis o copias de lecturas. La individuación de estos textos es la ímproba tarea asumida por el proyecto de investigación italiano La biblioteca ideale di Nietzsches, dirigido por Giuliano Campioni y Aldo Venturelli. Por medio de la investigación de las fuentes de Nietzsche (cuyos resultados se publican anualmente en el apartado «Quelle-Forschung» de los Nietzsche-Studien) se pretende, entre otras cosas, determinar qué textos de los cuadernos no son creación suya, y no reflejan por tanto ideas suyas, sino sus lecturas. Campioni reseña un caso flagrante de los malentendidos a los que puede conducir estas paráfrasis o resúmenes de sus lecturas: «El grado de reelaboración de ciertos temas, no ausentes en las obras publicadas, la relación entre estos apuntes de lectura y las afirmaciones de El anticristo y del Crepúsculo de los ídolos es un problema del intérprete, también 'grande' y filósofo: el lector ciertamente debe saber si lee una reflexión del aventurero diplomático francés Jacolliot, del filósofo Féré o de Tolstoi. Debe saber que cuando lee pasajes de tal crudeza fisiológica como: "La naturaleza no es inmoral cuando es despiadada con los degenerados" 6

5 G. Campioni, La biblioteca ideale di Nietzsche [en línea]. < http://www.cribecu.sns.it/ b31/nie.htm >.

6 Der Wille zur Macht, ed. E. Förster-Nietzsche, P. Gast y O. Weiß, en NietzscheWerke. Gesamtausgabe in Großoktav 2 Leipzig, Naumann/Kröner, 19 vols., 1894-1913, vols. XV y XVI, 1911, § 52, reeditado corregido y contextualizado en la lectura de la que forma parte como KSA 13, 15[41]. 
[...], se trata de apuntes de lectura y fieles paráfrasis de Charles Féré (Dégénérescence et criminalité. Essai phsysiologique, París, 1888, B. N., p. 104: "La nature est sans pitié pour les dégénerés; mais c'est à tort qu'on pourrait soutenir que la nature est insensible et inmorale")» 7 .

4) Apuntes de ideas divergentes o contrarias, cuando no pertenecen a la categoría anterior, sino que son planteamientos o ideas posibles que Nietzsche los imaginó y apuntó para tenerlos presente. La única manera de individuarlos es mediante el contexto y la comparación con los textos fidedignos. Dado el gusto experimentador y posibilista de Nietzsche, es importante que el intérprete tenga siempre presente esta posibilidad, y no se deje llevar alegremente por la 'novedad' de ciertos textos.

5) Apuntes de ideas no destinadas a la publicación. A primera vista, ante tantas dificultades ofrecidas por los póstumos, el criterio más lógico sería tomar como guía fundamental la obra publicada, y con ella interpretar la masa ingente de los póstumos. Pero la cuestión no es tan fácil, como han creído ciertos especialistas. Nietzsche dice expresamente que un número enorme de apuntes, de distintas épocas, los ha escrito 'para sí mismo', no en vista de ser publicados, porque contenían su pensamiento más propio sobre cuestiones arduas, que por el momento no tenía interés alguno en comunicarlo a los demás. Estos apuntes abarcan justamente las reflexiones más teoréticas, de manera que sobre su trasfondo descubrimos que las cortas pinceladas que aparecen en la obra publicada, no son caprichos resultantes de su incompetencia filosófica, sino alusiones calculadas, expresiones oblicuas, pero bien fundadas sobre una meditación prolongada e incansable. Este es el caso paradigmático de los fragmentos póstumos del otoñoinviero de 1887-1888, donde nos topamos con una declaración paradójica para quien ha empleado ya quince años de

7 G. Campioni, «"En el desierto de la ciencia”. Una nueva edición italiana de la Voluntad de poder de Nietzsche», Er, VIII 15 (1993), 215-39, p. 230. Campioni añade más ejemplos: los aforismos que aparecen en La voluntad de poder como si fueran de Nietzsche, $\S \S$ 748, 207, 179, 191, 718, 723, 759, 193, 224, 169, 335, 166, 723, 759, 193, 224 no son tales, sino paráfrasis o citas literales de Tolstoi. 
vida en una intensa actividad de escritor: «yo no presto ya atención a los lectores: ¿cómo podría escribir para lectores?... Pero me anoto a mí mismo, para mí mismo»8. (Es sorprendente que un especialista riguroso como Schlechta cayera en el desliz filológico de minimizar la importancia del legado póstumo, afirmando que no aportan nada esencial al conocimiento de Nietzsche, cuando abundan las declaraciones explícitas o indirectas del autor, tanto en la obra publicada como en los fragmentos póstumos 9 ). La única manera de individuar este tipo de apunte es basándose en declaraciones explícitas del autor, ampliando luego el campo por similitud. También es lícito dejar en esta categoría todos los apuntes que no hayan podido ser clasificados en las otras. La utilidad de estos textos es evidente: a veces surge la sospecha de si no contienen el pensamiento más propio de Nietzsche acerca de algunas cuestiones. Esta categoría de textos tiene una serie de consecuencias que hay que tratar en la siguiente aportación de la edición Colli-Montinari.

\section{LA ARTICULACIÓN OBRA PUBLICADA-APUNTES PÓSTUMOS}

Esa quinta categoría de textos indica la particular articulación que posee la obra nietzscheana, y que es otro descubrimiento aportado por la edición Colli-Montinari. La diferencia sustancial entre los apuntes no-publicados y los textos publicados reside en la enorme elaboración artística a la que han sido sometidos éstos. En los fragmentos póstumos hallamos numerosos apuntes que esbozan esa presentación artística y retórica. Los mismos contenidos aparecen en los fragmentos póstumos en su aspecto inmediato, tal cual los pensó Nietzsche, y en la obra publicada filtrados a través de su fino instinto artístico. Esta elaboración artística no puede entenderse sin recurrir al concepto fundamental nietzscheano de la 'máscara'10. Por medio de

8 «Ich achte die Leser nicht mehr: wie könnte ich für Leser schreiben?... Aber ich notire mich, für mich.» KSA 12, 9[188] p. 450.

9 Cf. en contraposición la opinión de un filólogo dotado de naturaleza filosófica: G. Colli, Scritti su Nietzsche, Milano, Adelphi, 19862, pp. 169-179.

10 No existe por ahora estudio más exhaustivo y extenso de la temática de la máscara, en sí misma, en conexión con el estilo nietzscheano y con el sentido de conjunto de su

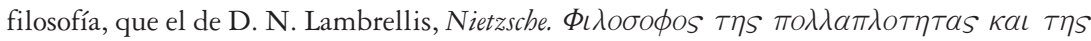


esa elaboración, Nietzsche 'escenifica teatralmente' los contenidos que él había descubierto en su interioridad de un modo inmediato. Pero es importante advertir que el sentido de la 'máscara' no es sólo ocultar, sino también mostrar. La máscara no oculta por miedo o por mala fe, porque no opone la ocultación a la 'mostración'; más bien, justamente deshace su oposición, vincula una a otra, crea las condiciones para que una genera a la otra. La máscara oculta ciertos aspectos, para poder mostrar mejor ciertos otros. La función básica de la máscara no es la ocultación de un mensaje o un sentido ideal, sino la separación, la generación de distancia, entre el autor y el texto, entre el texto y el lector, y por tanto entre el autor y el lector. Al interpretar un texto hay que tener presente esa función 'distanciadora' suya, porque con ella se dice mucho más de lo que 'parece'. El saber leer bien nietzscheano ${ }^{11}$ supone precisamente esto: un lector que advierta esta distancia, la asuma y sepa orientarse con ella en el laberinto nietzscheano. Para este lector atento, las máscaras son mucho más 'reveladoras' que la mayoría de las confesiones (Nietzsche insiste muchas veces sobre cuánta deshonestidad hay en las confesiones personales, y sobre todo cuánta mentira ante sí mismo). Así hay que acercarse a libros como Ecce homo, si no se quiere correr el riesgo de malentenderlos completamente. Por consiguiente, la elaboración artística a la que Nietzsche somete los textos no es en modo alguno un impedimento, sino una ayuda a la interpretación siempre que se sepa utilizarla. A este respecto, la comparación de los aforismos publicados con sus fragmentos póstumos paralelos ${ }^{12}$ es un recurso heurístico

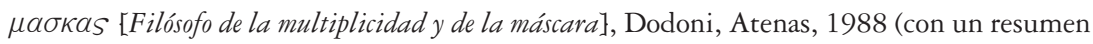
en inglés). Cf. también su artículo «The one and the many in Nietzsche», Nietzsche-Studien, 19 (1990), 129-142.

11 «La Filología, en efecto, es aquel arte honorable que exige a su cultivador ante todo una cosa: quedarse a un lado, permitirse tiempo, hacerse silencioso, hacerse lento..., como un arte y una pericia de orfebres de la palabra, que tiene que efectuar un trabajo finísimo y atento y no consigue nada si no lo consigue con un lento. [...] Para un arte tal no es tan fácil dejar lista cualquier cosa, él enseña a leer bien, es decir, a leer lentamente, en profundidad, mirando delante y detrás, con segundas intenciones, dejando las puertas abiertas, con dedos y ojos delicados... Pacientes amigos míos, este libro sólo desea para sí perfectos lectores y filólogos: ¡aprended a leerme bien!» Morgenröthe, Vorwort § 5, en KSA 3, p. 17

12 No con las redacciones previas, que sólo varían en mínimos aspectos formales, aunque en algún caso también puedan ser indicativas. 
muy efectivo. En resumen, esta articulación peculiar de la obra nietzscheana es otra aportación filosófica de la edición Colli-Montinari, que ha salido a la luz con la publicación de todos los manuscritos en su orden cronológico.

\section{ACABAmiento Del PENSAMiento NiETZSCHEANO}

La edición de una supuesta obra capital de Nietzsche, exponiendo su doctrina fundamental, la 'voluntad de poder', producía la impresión - y ha llegado a generar la 'ilusión'- de que Nietzsche poseía una doctrina acabada y cerrada acerca de la totalidad de todo lo existente: la naturaleza, el hombre, la sociedad, etc. Así se terminó por atribuirle, de manera inconsciente, un planteamiento dogmático; dogmatismo a la inversa, porque rechaza todos los dogmatismos tradicionales, pero dogmatismo al fin y al cabo. Éste es uno de lo eficaces reproches que Heidegger expresa de continuo casi subliminalmente en su monografía, sobre todo cuando contrapone esa 'nueva doctrina' a su 'camino del pensar'. Esta errónea visión del pensamiento nietzscheano tiene como consecuencia que se preste poca atención a la evolución de su pensamiento. Se piensa que su obra sólo ha evolucionado para alcanzar ese resultado capital y después sólo para perfilarlo. En realidad, cualquier lectura atenta de las obras nietzscheanas percibe claramente la enorme evolución que sufrió su pensamiento. La tasa de variación de su pensamiento es tan alta, que cualquier lector avezado siente la necesidad de centrar su estudio en una época de su producción, o recorrerlas poco a poco, antes de aventurarse a emitir juicios globales. Si existe un filosofar que se haya configurado, no en resultados y doctrinas, sino en un camino sin principio ni fin predeterminado, éste es más que ninguno el de Nietzsche.

\section{Filosofía experimental}

Con la edición Colli-Montinari tenemos acceso entonces, no sólo al 'taller', sino también al 'laboratorio' nietzscheano. Tras una lectura atenta se desprende una impresión de conjunto muy distinta a la que suscitaba La voluntad de poder. En vez del aspecto sentencioso y lapidario de su obra publicada, su pensamiento adquiere un aspecto 'experimental’ del que aquél es sólo una máscara más. A lo largo de los 
fragmentos póstumos asistimos a una inaudita puesta a prueba de ideas y conceptos, a una creación y un ensayo incesantes de conceptos, teorías y valoraciones. Comprendemos más que nunca la interpretación de Kaulbach de la filosofía nietzscheana como una 'filosofía experimental'. Descubrimos la actividad interior del pensamiento nietzscheano: ensayando nuevos conceptos, persigue neutralizar, como tales, todas nuestras convicciones más arraigadas, para no permitirse ni a sí mismo ni al lector, detenerse en ninguna tierra firme de la certeza. Por esa razón, las teorías que va formulando Nietzsche a lo largo de su trayectoria intelectual, no son doctrinas o dogmas, sino hipótesis y ensayos. La edición crítica ha servido para sacar a la luz el incesante movimiento de autosuperación al que se ve sometido el pensamiento nietzscheano. Teorías y valoraciones, basadas en puntos de vista específicos, se ven corregidas, rebatidas o superadas por otras perspectivas, que enriquecen nuestro conocimiento del fenómeno. Es un movimiento de auto-superación porque lo primero que Nietzsche somete a crítica incesantemente no son las creencias ajenas, sino sus propias convicciones; lo más importante no es superar las formas de pensar ajenas, por muy erróneas que sean, sino las propias, donde nos vemos atrapados y bloqueados ${ }^{13}$. En este sentido, la edición crítica nos revela que ningún pensador ha ejercitado y vivido tanto la autocrítica como Nietzsche. Y, sin embargo, éste aspecto quedaba muy oculto en las antiguas ediciones de su obra.

\section{Centralidad de la voluntad de POder}

En seguida se descubre que la presunta centralidad de la 'doctrina de la voluntad de poder' se contradice con todo lo anterior. En este movimiento de autosuperación que genera la filosofía experimental, la voluntad de poder es un concepto muy utilizado por su enorme fecundidad, pero no deja de ser un ensayo. Los fragmentos póstumos de la última época demuestran cómo Nietzsche lo sometió progresivamente a una crítica implacable, para depurarlo poco a poco, hasta

13 En esto consiste la 'pasión del conocimiento' que Nietzsche tematiza tanto a lo largo de su obra. Cf. sobra la temática M. Brusotti, Die Leidenschaft der Erkenntnis. Philosophie und ästhetische Lebensgestaltung bei Nietzsche von Morgenröthe bis Also sprach Zarathustra, Berlin, W. de Gruyter, 1997. 
llegar al punto de eliminarlo completamente, como consta en el controvertido apunte inédito: "Exotérico-esotérico. 1) Todo es voluntad contra voluntad. 2) No existe en absoluto ninguna voluntad. 1) Causalismo. 2) No existe nada parecido a causa-efecto» ${ }^{14}$. Así se explica su última renuncia a elaborar una obra sobre La voluntad de poder. Intérpretes anteriores a la edición Colli-Montinari, como Kaulbach, ya habían captado que la voluntad de poder no es la teoría central de su pensamiento. Por contra, todos aquellos que interpretaban el sentido global de su filosofía creyendo que la voluntad de poder era su concepto de fondo, quedan atrapados por un prejuicio. Tal es el caso de Heidegger (y no es el caso de Deleuze, aunque también sitúe en el centro de su interpretación la voluntad de poder, porque en realidad desde un principio ha partido siempre de la naturaleza 'nómada' del pensamiento nietzscheano). La publicación completa de los apuntes póstumos en su orden cronológico demuestra claramente que la voluntad de poder no fue su 'doctrina fundamental', sino sólo una hipótesis de cuyos defectos fue cada vez más consciente.

\section{FiLOSOFÍA y fiLOLOGÍA}

Si el Nietzsche de El nacimiento de la tragedia y de la segunda Intempestiva ponía en un conflicto irremediable filología y pensamiento, todo el Nietzsche posterior, a partir del gran giro de Humano, demasiado bumano, recuperaba la actividad filológica como componente imprescindible del desarrollo crítico y creativo del pensamiento. Baste recordar que una de las admoniciones del último Nietzsche, en vista del fortalecimiento de la cultura, era el 'aprender a leer bien'. La preocupación, el interés, el gusto por la realidad 'física' del texto supone al asunción de la esencial 'rugosidad' de lo real como condición básica de todo experimentar y crear. Ella explica que toda reconfiguración del corpus textual de un filósofo, con todo lo que incluye de cambios en las prácticas con las que el intérprete se enfrenta a esos textos, implica indefectiblemente el afloramiento progresivo de nuevas posibilidades de pensamiento, 'estrictamente filosóficas', que podríamos calificar, sin miedo a caer en viejos dogmatismos, como 'más ajustada' a la realidad multívoca y variante de la filosofía del autor. 